\title{
Anisotropic studies of quenched and indented potassium bromide cleavages
}

\author{
R RAVEENDRAN* and J R PANDYA ${ }^{\dagger}$ \\ Department of Physics, S. N. College, Kollam, Kerala 691001 , India \\ 'Department of Physics, Faculty of Science, M. S. University of Baroda, Vadodara 390002, \\ India \\ MS received 20 February 1995; revised 17 August 1996
}

\begin{abstract}
Kick's law and modified Kick's law for pyramidal indenter were used to study the mechanical response of indented and quenched potassium bromide cleavages. The study has disclosed an unusual interpretation of constants in the above laws. It suggested a pair of anisotropic indices which characterize a crystallographic direction. This study has also shown that there is atleast one crystallographic direction usually termed as homogeneous isotropic direction along which their mechanical properties are same. The implications are discussed in detail.
\end{abstract}

Keywords. Mechanical response; cleavage plane; pyramidal indenter.

\section{Introduction}

For studying mechanical response of indented surfaces, two empirical laws viz. Kick's law/Meyers law and modified Kick's law (Kick 1885; Meyer 1956; Hays and Kendal 1973) are available. Detailed study of the literature (Hanemann and Schultz 1941; Onitsch 1947; Grodzinski 1952; Westbrook and Conard 1973) indicates that the physical interpretations of constants in these laws are unsatisfactory. The aim of the present work is to critically re-examine these laws for the physical meanings of these constants by experimentally studying indented potassium bromide cleavages at room temperature, at elevated temperatures for different orientations in a phenomenological manner and by following the well known mathematical and graphical procedure. This is an extension of the earlier work carried out in our laboratory.

\section{Experimental}

Single crystals of potassium bromide grown from melt by Kyropoulos method (Kyropoulos 1963; Raveendran 1993) were used for indentation. Knoop indenter attached to C Z VERTIVAL microscope was used to indent for various applied loads $(1.25$ to $160 \mathrm{~g})$ and for fixed time of indentation $(15 \mathrm{sec})$. Approximately equal sized samples of freshly cleaved $\mathrm{KBr}$ faces-planes-having Miller indices (100) were selected. The indentation marks were produced for different orientations of the longer diagonal of the Knoop indenter with respect to direction [100] on $\mathrm{KBr}$ cleavages. These orientations were designated by angle $A$ between the reference direction [100] and the longer diagonal of the impression. For thermal treatment samples were kept in a muffle furnace, the temperature of which was gradually raised to the desired value and maintained at that value for $24 \mathrm{~h}$. It is known that if a brittle crystal is too much quenched to room temperature, it shatters into large number of pieces. To avoid this

*Author for correspondence 
the $\mathrm{KBr}$ crystals were quenched to room temperature by keeping the quenching rate as high as possible so that the samples could maintain their shapes and sizes. They were then cleaved at room temperature $\left(303^{\circ} \mathrm{K}\right)$. The cleaved specimen was kept on the stage of the vertival microscope. By considering the crystal edge which is along direction [001] as the reference direction, the orientation is determined by measuring the angle A between the longer diagonal of the indentation mark produced by the pyramidal knoop indenter on the cleavage surface and the direction [001]. This is done by carefully adjusting the rotation of the stage. Large number of thermally treated and untreated specimens were indented for orientations $0,10,20,30,40,50,60,70,80$ and $90^{\circ}$ and quenching temperatures $473,573,673,773$ and $873^{\circ} \mathrm{K}$.

\section{Results and discussion}

For pyramidal indenters, Kick's law is symbolically represented as

or

$$
p=a d^{n}
$$

$$
\log p=\log a+n \log d,
$$

where $p$ is the applied load, $d$ the diagonal length of the indentation mark $(\mu \mathrm{m})$ and $a$ and $n$ are constants of a given material where $a$ is the standard hardness and $n$ measure of the variation in hardness as a function of $p$ or $d$ or indicating capacity for work hardening (Mott 1956). The graphical analysis of (2) clearly indicated splitting of single straight line into two straight lines with slopes $n_{1}, n_{2}$ and intercepts $a_{1}, a_{2}$ for different crystals. The first line with $n_{1}$ and $a_{1}$ corresponds to low load region (LLR) and the second one with $n_{2}$ and $a_{2}$ to high load region (HLR) (Joshi and Pandya 1991; Jani et al 1994).

The present work also found the splitting of the above plot into two straight lines with $n_{1}, a_{1}$ and $n_{2}, a_{2}$ for cleavage faces of $\mathrm{KBr}$ crystals (Raveendran 1993). Due to visible scattering in $n$ and $a$ values, the necessity of application of modified Kick's law arises.

The modified Kick's law for pyramidal indenter is

or

$$
P-w=b d^{2},
$$

$$
\log P-w=\log b+2 \log d .
$$

Where $b$ is the intercept and $w$ the newtonian resistance pressure (Hays and Kendal 1973; Pratap and Hari Babu 1980; Joshi and Pandya 1991). The graphical plot of modified Kick's law also shows splitting of $\log (P-w)$ vs $\log d$ into two recognizable straight lines with intercepts $b_{1}, b_{2}$ and newtonian resistance pressures $w_{1}$ and $w_{2}$. It is well recognized that for single crystals of different types (Berzina et al 1965; Joshi and Pandya 1993) in HLR hardness is constant and independent of applied load. Hence in the present work for Kick's law and modified Kick's law, only HLR is considered for straight line plots with $n_{2}, a_{2}$ and $b_{2}, w_{2}$ parameters vary with ' $A$ ' for different temperatures (table 1).

\subsection{Variation of anisotropic indices with orientation}

A careful study of plots of $n_{2}$ vs $A, a_{2}$ vs $A$ and $b_{2}$ vs $A$ for room temperature and different quenching temperatures (figures $1-3$ ) reveals the following: (i) all these plots 
Table 1. Angle A slopes $n_{20 b s}, n_{2 \bmod }$, intercepts $a_{2}, b_{2}$ and newtonian resistance pressure $w_{2}$ of the plots of $\log d$ vs $\log P$ for $\mathrm{KBr}$ at room temperature $303^{\circ} \mathrm{K}$ and quenching temperatures $673^{\circ} \mathrm{K}$ and $873^{\circ} \mathrm{K}$.

\begin{tabular}{|c|c|c|c|c|c|}
\hline Angle A & $n_{\text {2obs. }}$ & $n_{2 \bmod }$ & $w_{2}$ & $b_{2} \times 10^{-3}$ & $a_{2} \times 10^{-3}$ \\
\hline \multicolumn{6}{|c|}{ Room temperature $\left(303^{\circ} \mathrm{K}\right)$} \\
\hline 0 & $1 \cdot 8500$ & $2 \cdot 1460$ & 1.6167 & 0.5939 & 1.5759 \\
\hline 10 & $1 \cdot 8000$ & $2 \cdot 0000$ & 1.9975 & 0.5635 & 2.0345 \\
\hline 20 & 1.8400 & $2 \cdot 0000$ & 1.5165 & 0.5314 & $1 \cdot 5028$ \\
\hline 30 & $1 \cdot 7910$ & 1.9985 & 3.0851 & 0.4860 & 1.7740 \\
\hline 40 & $1 \cdot 7516$ & 1.9900 & $2 \cdot 6160$ & 0.4411 & $2 \cdot 2030$ \\
\hline 50 & 1.7476 & $2 \cdot 0002$ & $2 \cdot 1178$ & 0.4452 & $2 \cdot 2045$ \\
\hline 60 & 1.7756 & 1.9817 & $2 \cdot 3431$ & 0.4920 & $2 \cdot 9625$ \\
\hline 70 & $1 \cdot 8661$ & 1.9990 & 2.6178 & 0.5353 & $1 \cdot 2705$ \\
\hline 80 & $1 \cdot 8674$ & $2 \cdot 0102$ & $3 \cdot 1518$ & 0.5662 & $1 \cdot 3822$ \\
\hline 90 & 1.8644 & $2 \cdot 0000$ & 1.6597 & 0.6055 & 1.4473 \\
\hline \multicolumn{6}{|c|}{ Quenching temperature $\left(673^{\circ} \mathrm{K}\right)$} \\
\hline 0 & $1 \cdot 8310$ & $2 \cdot 0000$ & $1 \cdot 1585$ & 0.5620 & $1 \cdot 7128$ \\
\hline 10 & $1 \cdot 8500$ & $2 \cdot 0101$ & $1 \cdot 3900$ & 0.5142 & 1.4196 \\
\hline 20 & 1.8308 & 1.9910 & 1.8518 & 0.5081 & 1.5305 \\
\hline 30 & $1 \cdot 8000$ & $2 \cdot 0000$ & $2 \cdot 0180$ & 0.4311 & 1.6943 \\
\hline 40 & 1.7791 & $2 \cdot 0000$ & $2 \cdot 5000$ & 0.4296 & 1.7284 \\
\hline 50 & 1.7791 & 2.0110 & $2-1916$ & 0.4042 & 1.7301 \\
\hline 60 & 1.8010 & 1.9900 & $2 \cdot 7500$ & 0.4311 & 1.6422 \\
\hline 70 & 1.8410 & $2 \cdot 1040$ & 1.9516 & $0 \cdot 4861$ & 1.4504 \\
\hline 80 & 1.8586 & 2.0000 & 1.8616 & 0.5190 & 1.3394 \\
\hline 90 & $1 \cdot 8310$ & $2 \cdot 0000$ & $1 \cdot 1690$ & 0.5541 & 1.7128 \\
\hline \multicolumn{6}{|c|}{ Quenching temperature $\left(873^{\circ} \mathrm{K}\right)$} \\
\hline 0 & 1.8715 & 2.0000 & $1 \cdot 3251$ & 0.7460 & $1 \cdot 1555$ \\
\hline 10 & 1.8281 & 2.0000 & $2 \cdot 0500$ & 0.7254 & 2.0927 \\
\hline 20 & 1.7917 & 1.9990 & $2 \cdot 1600$ & 0.6920 & $2 \cdot 4482$ \\
\hline 30 & 1.7784 & 2.0000 & $2 \cdot 8500$ & 0.6395 & $2 \cdot 5604$ \\
\hline 40 & $1 \cdot 7680$ & 2.0101 & $2 \cdot 9000$ & 0.6017 & $2 \cdot 6600$ \\
\hline 50 & $1 \cdot 7680$ & $2 \cdot 0101$ & 3.0030 & 0.6017 & $2 \cdot 6600$ \\
\hline 60 & $1 \cdot 7891$ & 2.0000 & $2 \cdot 0850$ & 0.6491 & 2.4558 \\
\hline 70 & $1 \cdot 7990$ & 1.9990 & $2 \cdot 1050$ & 0.7248 & $2 \cdot 3535$ \\
\hline 80 & 1.8391 & 1.9616 & 1.9616 & 0.7410 & 1.9925 \\
\hline 90 & 1.8685 & 2.0000 & 2.0000 & 0.7460 & $1 \cdot 7836$ \\
\hline
\end{tabular}

have one common feature, viz. $n_{2}, a_{2}$ and $b_{2}$ have extremum values for orientation $45^{\circ}$ and for all temperatures. This orientation corresponds to direction [110] on the surface, (ii) these values are different for room temperature and different quenching temperatures and (iii) $n_{2}$ has a minimum value for $A=45^{\circ}$ (figure 1) whereas $a_{2}$ has a maximum value for the same orientation (figure 2).

This is understandable as in HLR hardness is constant and independent of applied load. Hence with a decrease of $n_{2}$, there is a corresponding increase for $a_{2}$. Similarly the same relation holds for plot of $b_{2}$ vs $A$ (figure 3) and approximately for $w_{2}$ vs $A$ (not shown). The approximation in the plot of $w_{2}$ vs $A$ is due to the fact that in (3) $w$ is assumed to be newtonian resistance pressure which does not allow plastic deformation (Hays and Kendal 1973). It has units whereas $n_{2}$ is a slope and a pure number. 


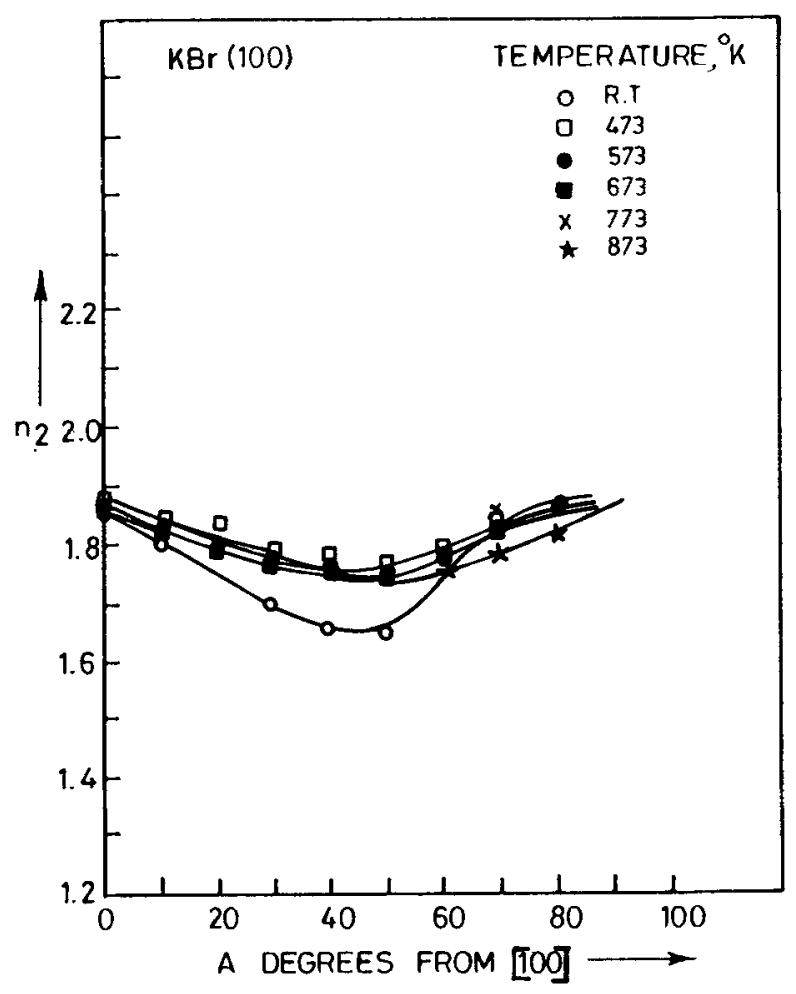

Figure 1. Plot of $n_{2}$ vs $A$ for $\mathrm{KBr}$ cleavage plane (100) at different temperatures.

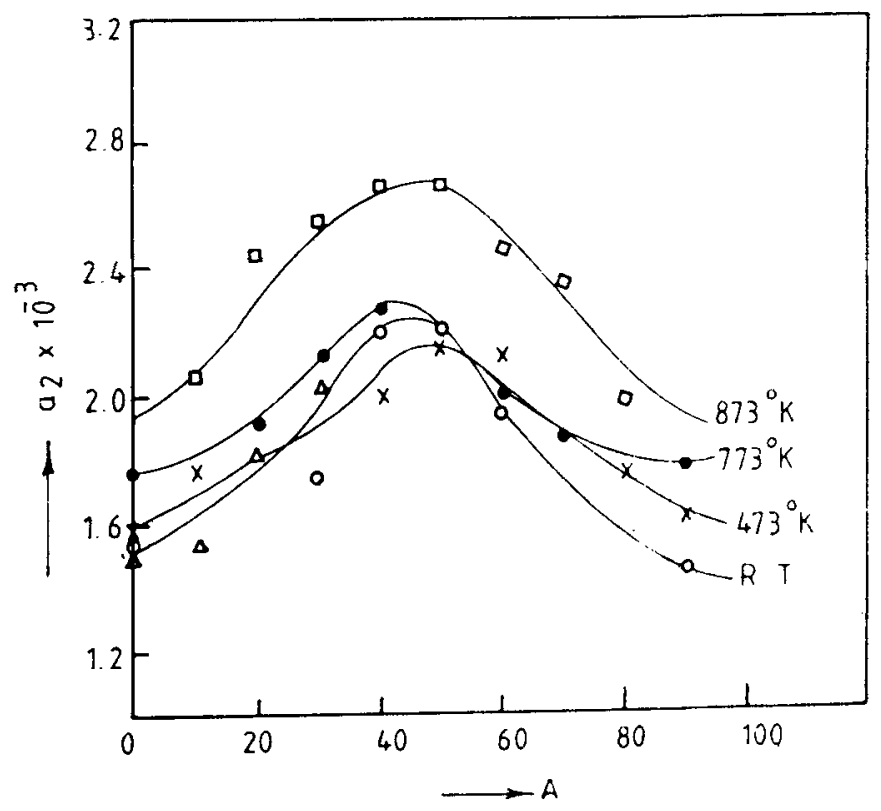

Figure 2. Plot of $a_{2}$ vs $A$ for $\mathrm{KBr}$ cleavage plane (100) at different temperatures. 


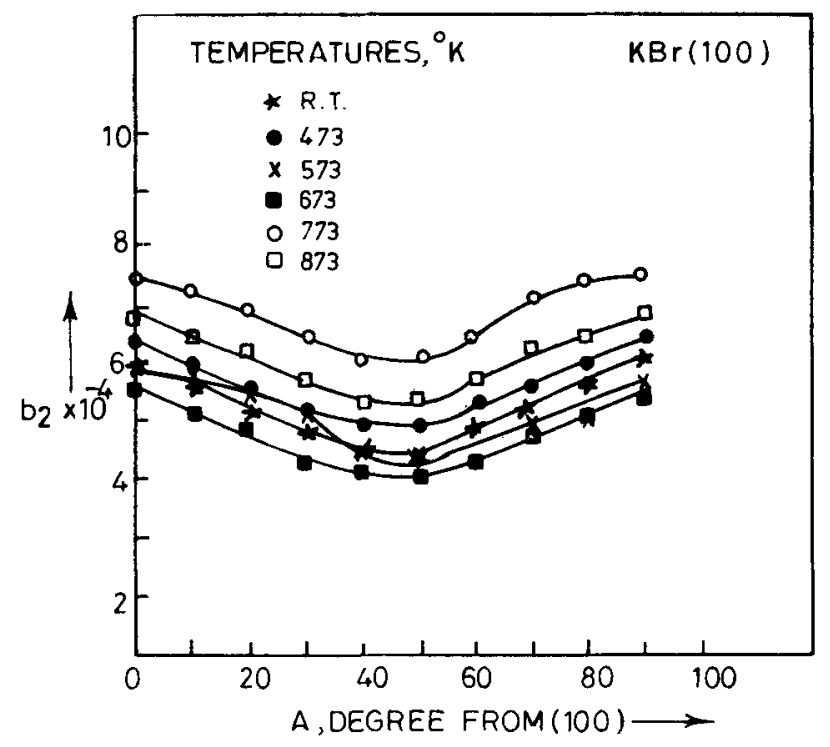

Figure 3. Plot of $b_{2}$ vs $A$ for $\mathrm{KBr}$ cleavage plane (100) at different temperatures.

It is thus clear from the above analysis that the direction [110] corresponding to $45^{\circ}$ orientation on cleavage faces of this crystal occupies an important position in the study of Kick's law and modified Kick's law.

\subsection{Variation of anisotropic indices with temperature}

It is found that for this crystal, the plots of $\log a_{2} T_{\mathrm{q}}$ vs $\log T_{\mathrm{q}}$ (figure 4) and $\log b_{2} T_{\mathrm{q}}$ vs $\log T_{\mathrm{q}}$ (not shown) for different orientation consists of a series of almost parallel straight lines with almost equal slopes and different intercepts corresponding to different orientations. The plots follow general equation

$$
\log g T_{\mathbf{q}}=m \log T_{\mathbf{q}}+\log C r .
$$

Where the variable $g$ may be $a_{2}$ or $b_{2}$ and the straight line has slope $m$ and intercept $C r$ where $r$ denotes a given orientation. Different values of $r$ gives a series of parallel lines.

Simplification of the above yields

$$
g T_{\mathrm{q}}^{\mathbf{1}-m r}=\mathrm{Cr} .
$$

Replacement of $g$ by $a_{2}$ and $b_{2}$ in the above yields two equations

and

$$
a_{2} T_{\mathrm{q}}^{1-m^{2}}=\mathrm{Cr}_{2}
$$

$$
b_{2} T_{q}^{1-m 3}=C r_{3} .
$$

The relation between $w_{2}$ and $T_{\mathrm{q}}$ is not as simple as the one given above between $a_{2}$ (or $b_{2}$ ) with $T_{\mathrm{q}}$ due to the reasons mentioned above.

It should be noted that $K_{2}$ and $K_{3}$ are independent of quenching temperatures but depend on orientation A. $K_{2}$ and $K_{3}$ are less than unity and negative and they vary 


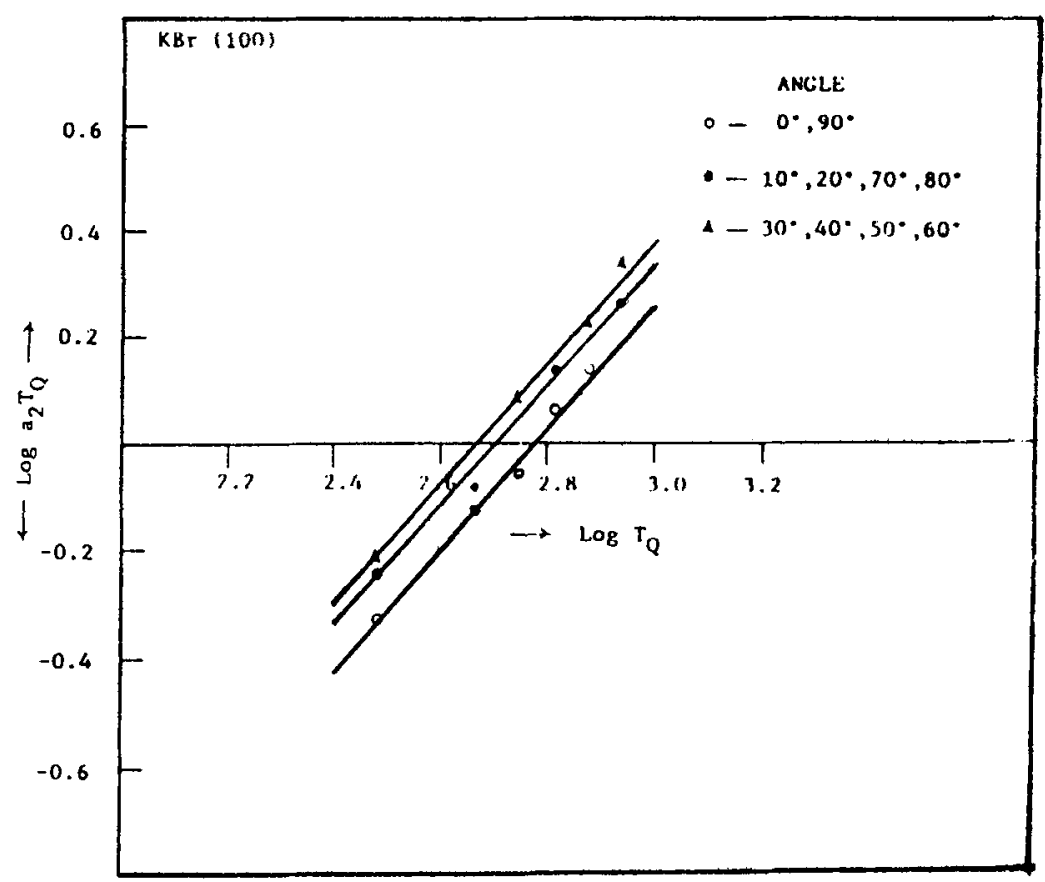

Figure 4. Plot of $\log a_{2} T_{q} v \log T_{q}$ for different orientations on $\mathrm{KBr}(100)$ cleavages.

Table 2. Values of slope $m_{2}$, intercept $C r_{2}$ and $K_{2}\left(=1-m_{2}\right)$ from the plot of $\log { }^{\prime} a_{2}{ }^{\prime} T_{\mathrm{q}}$ vs $\log T_{\mathrm{q}}$.

\begin{tabular}{|c|c|c|c|}
\hline $\begin{array}{l}\text { Orientation } \\
\mathrm{A}(\mathrm{deg})\end{array}$ & $\begin{array}{c}\text { Slope } \\
m_{2}\end{array}$ & $\begin{array}{c}\text { Intercept } \\
C r^{2} \\
\left(\times 10^{-3}\right)\end{array}$ & $\begin{array}{l}1-m_{2} \\
=K_{2}\end{array}$ \\
\hline 0 & $1 \cdot 125$ & 0.750 & -0.125 \\
\hline 10 & 1.071 & $1 \cdot 311$ & -0.071 \\
\hline 20 & $1-071$ & $1 \cdot 311$ & -0.071 \\
\hline 30 & 1.000 & $2 \cdot 290$ & +0.000 \\
\hline 40 & 1.000 & $2 \cdot 290$ & +0.000 \\
\hline 50 & 1.000 & $2 \cdot 290$ & +0.000 \\
\hline 60 & 1.000 & $2 \cdot 290$ & +0.000 \\
\hline 70 & 1.071 & $1 \cdot 311$ & -0.072 \\
\hline 80 & $1 \cdot 071$ & 1.311 & -0.071 \\
\hline 90 & $1 \cdot 125$ & 0.750 & -0.125 \\
\hline
\end{tabular}

from -0.125 to 0 and -0.25 to 0 respectively. Similarly the values of $\mathrm{Cr}_{2}$ and $\mathrm{Cr}_{3}$ are also independent of temperature but depends on orientation. They also vary from 0.75 to 2.29 and 1.33 to 5.62 respectively. The observation in tables 2 and 3 indicate that the direction [110] is important. The present analysis has convincingly shown that $n$ and $a$ in Kick's law or $b$ and $w$ in modified Kick's law are not simple constants characteristic of the materials but are constants with a deep meaning. They depend on orientation of the pyramidal indenter with respect to a choosen direction on a crystal face and temperature. 


\begin{tabular}{|c|c|c|c|}
\hline $\begin{array}{l}\text { Orientation } \\
\text { A(deg) }\end{array}$ & $\begin{array}{l}\text { Slope } \\
m_{3}\end{array}$ & $\begin{array}{c}\text { Intercept } \\
\mathrm{Cr}_{3}\end{array}$ & $\begin{array}{l}1-m_{3} \\
=K_{3}\end{array}$ \\
\hline 0 & 1.25 & $1 \cdot 33$ & $-0 \cdot 25$ \\
\hline 10 & $1 \cdot 20$ & 1.73 & $-0 \cdot 20$ \\
\hline 20 & $1 \cdot 14$ & $2 \cdot 26$ & $-0 \cdot 14$ \\
\hline 30 & 1.00 & $5 \cdot 62$ & +0.00 \\
\hline 40 & 1.00 & $5 \cdot 12$ & +000 \\
\hline 50 & 1.00 & $5 \cdot 12$ & +0.00 \\
\hline 60 & 1.00 & 5.62 & -0.00 \\
\hline 70 & $1 \cdot 14$ & $2 \cdot 26$ & $-0 \cdot 14$ \\
\hline 80 & $1 \cdot 20$ & 1.73 & -0.20 \\
\hline 90 & 1.25 & $1 \cdot 33$ & -0.25 \\
\hline
\end{tabular}

\subsection{Importance of direction [110]}

Interesting and useful conclusions drawn from the above analysis is that the direction [110] for cleavage face of $\mathrm{KBr}$ in particular and the family of direction $\langle 110\rangle$ in general is the one along which the quantities characterizing straight lines viz. $\left(n_{2}, a_{2}\right)$ and $\left(b_{2}, w_{2}\right)$ have extremum values. The orientation of the indenter corresponding to this direction is $45^{\circ}$ with respect to reference direction [110] in particular and $\langle 110\rangle$ in general. From the crystal structure of $\mathrm{KBr}$ (not shown), it is clear that the projection of direction [111] on $\mathrm{KBr}$ cleavage surface (100) is [110]. Further the arrangements of ions along this direction and direction [111] are the arrays of like ions separated by a constant distance and facing arrays of unlike ions on all sides. Since the ionic charges are identical (positive or negative) there is repulsion among them along these directions. If this is the only factor in the lattice, the repulsive force will destory it. However, there are attractions due to the proximity of ions of opposite charges. Hence on the whole the lattice is maintained. However, the value of a property along this direction severely affected due to the arrays of like charges. According to Kick's law for a constant $P$ and $d$, if $n$ decreases $a$ must increase so that the product ad $^{n}$ has a constant value $(P)$ provided $P$ and $d$ are kept constant. However the physical meaning of $a$ is much different from $n$. In the literature $a$ is described as standard hardness. On the basis of the current analysis it is felt that the use of the above phrase is a misnomer. It should be considered as a constant dependent on direction and temperature and the minimum value of $n$ and maximum value of $a$ are independent of direction and temperature. In the HLR along direction [110] the minimum value of $n_{2}$ corresponds with a maximum value of $a_{2}$ at all quenching temperatures and room temperature. In the present analysis weightage is given to HLR region in view of the fact that hardness is constant and independent of applied loads.

In the modified Kick's law pair of important quantities are $b$ and $w$. The meaning given by Hays and Kendal (1973) for these quantities are different. $b$ is considered as standard hardness and $w$ the Newtonian resistance pressure responsible for the elastic deformation. However the present analysis shows that these quantities are dependent on the direction along which they are determined i.e. the crystal structure. Further only along direction [110], $b_{2}$ and $w_{2}$ have extremum values and are independent of 
temperature and direction. Since along this direction the quantities $\left(n_{2}, a_{2}\right)$ and $\left(b_{2}, w_{2}\right)$ are independent of direction, this direction can be considered as homogeneous and isotropic direction. Hence it should be taken as reference direction and not the direction [100]. Further, it is well known that all real crystals contain imperfections. The above analysis has taken into account only crystal structure and not impurities and imperfections of different types in the crystals.

\section{Conclusions}

The following conclusions are drawn from the experimental study of the variation of applied load with longer diagonal of the Knoop indentation mark for different orientations and quenching temperatures.

(I) The plots of $\log d$ vs $\log p$ consist of two clearly recognizable straight lines having different slopes $\left(n_{1}\right.$ and $\left.n_{2}\right)$ and different intercepts $\left(a_{1}\right.$ and $\left.a_{2}\right)$.

(II) The variation in the exponents of $d$ can be eliminated by employing modified Kick's law, where the exponent is nearly equal to 2 and $n_{2}$ and $a_{2}$ is replaced by $b_{2}$ and $w_{2}$. This law is applicable only in HLR.

(III) $\left(n_{2}, a_{2}\right)$ and $\left(b_{2}, w_{2}\right)$ have clear relation with orientation $\mathrm{A}$ and quenching temperatures and room temperature. Along [110] or in general $\langle 110\rangle$ for $\mathrm{KBr}$ crystals extremum values which are independent of orientation and temperature.

and $a_{2}$ and $b_{2}$ depend on quenching temperature and in HLR follow the relations

$$
a_{2} T_{\mathrm{q}}^{1-m 2}=\text { constant, }
$$

$$
b_{2} T_{\mathbf{q}}^{1-m 3}=\text { constant. }
$$

\section{References}

Berzina I G, Berman I B and Savintsev P A 1965 Sov. Phys. Crystallogr. 4483

Grodzinski P 1952 Schweiz arch angew Wiss 18282

Hanemann H and Schultz F 1941 Metalkde 33275

Hays C and Kendal E G 1973 Metallography 6275

Jani T M, Pandya G R and Desai C F 1994 Cryst. Res. Technol. 29 K3

Joshi D R and Pandya J R 1991 Cryst. Res. Technol. 26433

Joshi D R and Pandya J R 1993 Cryst. Res. Technol. 28103

Kick F 1885 Das Gastez des proportionalen Widerstandes und Anwendung (ed.) L Felix (Leipzig)

Kyropoulos S 1963 in Art and science of growing crystals (ed.) J Gilman (New York: John Wiley and Sons Inc) p. 413

Meyer L 1956 in Microindentation hardness testing (London: Butterworths Scientific publications,) Ch. 1 Mott B W 1956 Microindentation hardness testing (London: Butterworths Scientific publication) Ch. 1 Onitsch E M 1947 Mikroskopie 2131

Pratap K J and Hari Babu V 1980 Bull. Mater. Sci. 243

Raveendran R 1993 Ph. D. Thesis, M.S. University of Baroda, Baroda

Westbrook J H and Conard H 1973 The science of hardness and its research applications (Óhio: American Society for Metals) 